\title{
A three-dimensional comparison of a morphometric and conventional cephalometric midsagittal planes for craniofacial asymmetry
}

\author{
Janalt Damstra - Zacharias Fourie - Marnix De Wit • \\ Yijin Ren
}

Received: 23 August 2010 / Accepted: 9 January 2011 /Published online: 27 January 2011

(C) The Author(s) 2011. This article is published with open access at Springerlink.com

\begin{abstract}
Morphometric methods are used in biology to study object symmetry in living organisms and to determine the true plane of symmetry. The aim of this study was to determine if there are clinical differences between three-dimensional (3D) cephalometric midsagittal planes used to describe craniofacial asymmetry and a true symmetry plane derived from a morphometric method based on visible facial features. The sample consisted of 14 dry skulls ( 9 symmetric and 5 asymmetric) with metallic markers which were imaged with conebeam computed tomography. An error study and statistical analysis were performed to validate the morphometric method. The morphometric and conventional cephalometric planes were constructed and compared. The 3D cephalometric planes constructed as perpendiculars to the Frankfort horizontal plane resembled the morphometric plane the most in both the symmetric and asymmetric groups with mean differences of less than $1.00 \mathrm{~mm}$ for most variables. However, the standard deviations were often large and clinically significant for these variables. There were clinically relevant differences $(>1.00 \mathrm{~mm})$ between the different 3D cephalometric midsagittal planes and the true plane of symmetry determined by the visible facial features. The difference between 3D cephalometric midsagittal planes and the true plane of symmetry determined by the visible facial features were clinically relevant. Care has to be taken
\end{abstract}

J. Damstra $(\bowtie) \cdot$ Z. Fourie $\cdot$ M. De Wit $\cdot$ Y. Ren Department of Orthodontics, University Medical Center Groningen, University of Groningen,

Po. Box. 30.001, Groningen 9700 RB, The Netherlands

e-mail: j.damstra@dmo.umcg.nl using cephalometric midsagittal planes for diagnosis and treatment planning of craniofacial asymmetry as they might differ from the true plane of symmetry as determined by morphometrics.

Keywords Orthodontics · Three-dimensional (3D) . Cephalometry Craniofacial asymmetry · Midsagittal plane . Morphometrics $\cdot$ Procrustes analysis

\section{Introduction}

In biology, bilateral symmetry is described as matching symmetry or object symmetry [1]. Matching symmetry refers to a structure of interest which is present in two separate copies of a mirror image of one another, each located on either side of the body. With object symmetry, the structure is symmetric within itself and therefore has an internal plane of symmetry so that the left and right halves are mirror images of each other. The human skull is an example of object symmetry. Therefore, clinical diagnosis and treatment planning in orthodontics and maxillofacial surgery of craniofacial asymmetry are reinforced by measurements made to an internal symmetry plane or midsagittal plane [2-7]. The symmetry plane of the skull is also fundamental in other areas of medicine, for example the study of functional and anatomical brain symmetry $[8,9]$.

In the literature, there is no consensus to which is the best or most accurate cephalometric plane to describe craniofacial asymmetry. Most cephalometric 3D analyses rely on midsagittal planes based on midline structures [4-7, 10, 11]. This has evoked some concerns regarding the validity of these reference planes because living organisms are hardly ever perfectly symmetric and a degree of facial 
asymmetry is a common phenomenon in nature $[1,12,13]$. The structures that lie in the midsagittal plane of the ideal body plan might also be affected. In other words, the surfaces containing the midline points can deviate from the true plane of symmetry. It has been suggested that internal structures of the skull are irrelevant to the visible facial symmetry, i.e., the midline of the cranial base can deviate from the visible facial symmetry [15]. Although this is true for patients with pathological asymmetries for example plagiocephaly or hemifacial microsomia, it may also apply to other asymmetries and even symmetric skulls. Kwon et al. [7] studied a group of noncleft or plagiocephaly patients and found no difference in the cranial base between the symmetric and asymmetric groups and concluded that the cranial base structures were not dominant factors in explaining the degree of facial asymmetry. Provided that the internal structures of the skull are relevant to visual symmetry perception, this result suggests that the cranial base may be used as reference to determine the midsagittal plane for mild or moderate craniofacial asymmetries.

To overcome the possible problems of cephalometric midsagittal planes, shape analysis by means of morphometric methods such as Procrustes analysis and Euclidean distance matrix analysis has been applied to study craniofacial asymmetry $[14,16-20]$. Morphometric methods are accepted in all fields of biology to determine the true plane of symmetry in structures with object symmetry [1]. Since the perception of symmetry of faces is very important [20], the midsagittal plane used for diagnosis and treatment planning should be determined by the external visible facial features [16]. Landmark or surface-based Procrustes analysis uses visible facial features as reference to align original and mirrored images and therefore determines the true plane of symmetry relevant to facial perception. This morphometric approach has been shown to produce very accurate and reliable midsagittal planes that can be used for comparative study [15, 18-20].

Unfortunately, the extraction of the midsagittal plane by means of morphometrics requires additional training, additional software, and could be more costly which limits its clinical use. This might explain why 3D evaluation of craniofacial symmetry is still primarily performed with cephalometric methods [2-7]. However, these cephalometric planes might differ from the true plane of symmetry providing unreliable or even misleading information for the diagnosis and treatment planning.

Therefore, the aim of this study was to investigate if the cephalometric midsagittal planes using internal and midline structures are relevant to visible facial symmetry. Six cephalometric midsagittal planes described in the literature [2-7] were compared to the true plane of symmetry determined by morphometrics using visible facial features rather than internal landmarks as reference.

\section{Materials and methods}

\section{Materials}

To reduce random error due to landmark identification, dry human skulls were used with radiopaque metal markers. The sample was selected from a collection of anonymous dry skulls from the Department of Orthodontics of the University Medical Center Groningen. No ethical approval was required. Two groups of skulls were studied: (1) a group with visible asymmetry and (2) a group with no visible asymmetry. Before the study sample was selected, the anatomical landmarks described in Table 1 were marked on the skulls with a pencil by means of consensus of two observers (JD and ZF). Visible deviation was defined as at least 4-mm deviation of menton (Me) from the midline [6]. A midline was constructed with a laser beam passing through nasion $(\mathrm{N})$ and the anterior nasal spine (ANS). For inclusion in the symmetric group, deviation of less than $4 \mathrm{~mm}$ of Me from the constructed laser beam midline was a criterion. Because the growth of cranial base could be affected in subjects with congenital asymmetry, skulls with a cleft palate, hemifacial microsomia, or plagiocephaly were not considered. In both groups, the skulls also had to have a fixed occlusion, with the mandible fixed to the skull by means of two metal springs. Ultimately, a total of 14 skulls (5 asymmetric and 9 symmetric) were included for this study. Prior to the radiographic examination, metal markers with a diameter of $1.5 \mathrm{~mm}$ were glued onto the selected landmarks (Table 1) with cyanoacrylate glue (Unirapide Gold; Pattex, Henkel, Nieuwegein, Netherlands).

The cone-beam computed tomography (CBCT) images were acquired with the KaVo 3D eXam scanner (KaVo Dental $\mathrm{GmbH}$, Bismarckring, Germany). The skulls were placed in the CBCT scanner with the Frankfort horizontal (FH) plane parallel to the floor and the midline laser beam of the CBCT scanner passing through $\mathrm{N}$. The skulls were scanned with a 0.30 -voxel size resolution ( $120 \mathrm{kV}, 37.07 \mathrm{~mA} \mathrm{~s}$, and $26.9 \mathrm{~s})$. The CBCT datasets were exported from the eXamVisionQ (Imaging Sciences International LCC, Hatfield, PA, USA) software in DICOM multi-file format and imported into the SimPlant ${ }^{\circledR}$ Ortho Pro 2.1 software (Materialise Dental, Leuven, Belgium). Surface models for suitable visualization of the hard tissues were created, and the landmarks described in Table 1 were digitized using the middle of the metal markers as reference.

After the landmarks were digitized, the software automatically constructed the cephalometric 3D midsagittal planes (Table 1) commonly used for 3D cephalometric assessment of craniofacial asymmetry in maxillofacial surgery planning $[4-7,10,11]$. These planes were divided into two groups according to the method of construction, $i$. e., by connection of three midline structures $(1,2$, and 3$)$ or 
Table 1 Landmarks and cephalometric planes used in this study

\begin{tabular}{|c|c|c|}
\hline Landmarks & Abbreviation & Definition \\
\hline \multicolumn{3}{|l|}{ Unilateral } \\
\hline Sella & $\mathrm{S}$ & $\begin{array}{l}\text { Center of the sella turcica (in this case the marker was placed in the center of the } \\
\text { floor of sella turcica) }\end{array}$ \\
\hline Nasion & $\mathrm{N}$ & Most anterior of the frontonasal suture in the median plane \\
\hline Basion & $\mathrm{Ba} / \mathrm{MDFM}$ & Middorsal point of the anterior margin of the foramen magnum \\
\hline Point A & A & $\begin{array}{l}\text { Point at the deepest midline concavity on the maxilla between the anterior nasal } \\
\text { spine and prosthion }\end{array}$ \\
\hline Pogonion & Pog & Most anterior point of the bony chin in the median plane \\
\hline Menton & $\mathrm{Me}$ & Most inferior part of the bony chin in the median plane \\
\hline Upper incisor contact & $\mathrm{UI}_{\text {contact }}$ & Contact point of the two central upper incisor teeth \\
\hline Lower incisor contact & $\mathrm{LI}_{\text {contact }}$ & Contact point of the two central lower incisor teeth \\
\hline \multicolumn{3}{|l|}{ Bilateral } \\
\hline Orbitale & Or & Lowest point in the inferior margin of the orbit \\
\hline Supraorbital foramen ${ }^{\mathrm{a}}$ & SOF & Midpoint of the supraorbital foramen \\
\hline Medial zygomaticofrontal suture ${ }^{\mathrm{a}}$ & MZF & Medial point on the orbital rim of the zygomaticofrontal suture \\
\hline Frontonasomaxillare $^{\mathrm{a}}$ & FNM & Intersection of the nasomaxillary, frontomaxillary, and frontonasal sutures \\
\hline Fontorbitomaxillare $^{\mathrm{a}}$ & FOM & Lateral point of the frontomaxillary suture on the medial margin of the orbit \\
\hline Foramen spinosum & $\mathrm{FSp}$ & Center of the foramen spinosum \\
\hline Porion & Po/SLEAM & Superior lateral point of the external auditory meatus \\
\hline Jugulare & $\mathrm{J}$ & Intersection of the outline of the maxillary tuberosity and the zygomatic buttress \\
\hline First upper molar & U6 & Tip of the mesiobuccal cusp of the maxillary first permanent molar \\
\hline First lower molar & L6 & Tip of the mesiobuccal cusp of the mandibular first permanent molar \\
\hline Gonion & Go & $\begin{array}{l}\text { Point at each mandibular angle defined by dropping a perpendicular from the } \\
\text { intersection point of the tangent lines to the posterior margin of the ramus and } \\
\text { the inferior margin of the mandibular body }\end{array}$ \\
\hline Zygomatic arch point & $\mathrm{Za}$ & Most lateral border of the zygomatic arch \\
\hline Anterior clinoid process & $\mathrm{ACP}$ & Tip of the anterior clinoid process \\
\hline Lateral foramen magnum & LFM & Most lateral point of the foramen magnum \\
\hline \multicolumn{3}{|l|}{ Generated by computer software } \\
\hline Anterior clinoid midpoint & $\mathrm{ACP}_{\text {midpoint }}$ & Midpoint between the left and right ACP \\
\hline Foramen spinosum midpoint & ELSA & Midpoint between the left and right FSp \\
\hline Foramen magnum midpoint & LFM $_{\text {midpoint }}$ & Midpoint between the left and right LFM \\
\hline Porion midpoint & $\mathrm{Po}_{\text {midpoint }}$ & Midpoint between the left and right Po \\
\hline 3D midsagittal planes through three & midline struc & \\
\hline Plane passing th & rough points $S$ & and ANS \\
\hline Plane passing th & $\operatorname{arough} \mathrm{S}, \mathrm{N}$, an & \\
\hline $\begin{array}{l}\text { Plane passing th } \\
\text { anterior clinoic }\end{array}$ & $\begin{array}{l}\text { arough the mid } \\
\text { d processes anc }\end{array}$ & between the most lateral points on the foramen magnum, the midpoint between the \\
\hline 3D midsagittal planes through two & midline struct & and perpendicular to a horizontal plane \\
\hline $\begin{array}{l}\text { Plane passing th } \\
\text { landmarks of } C\end{array}$ & $\begin{array}{l}\text { Irough points } \mathrm{S} \\
\text { Or and } \mathrm{Po}_{\text {midpo }}\end{array}$ & and perpendicular to the Frankfort horizontal plane (FH passing through the bilateral \\
\hline $\begin{array}{l}\text { Vertical plane pa } \\
\text { perpendicular } t\end{array}$ & $\begin{array}{l}\text { assing through } \\
\text { to the FH plan }\end{array}$ & uperior point of the $\mathrm{Cg}$, a midpoint between the two anterior clinoid processes and \\
\hline $\begin{array}{l}\text { Plane passing th } \\
\text { points of SLE }\end{array}$ & $\begin{array}{l}\text { rough ELSA, } \\
\text { AM and point }\end{array}$ & $\begin{array}{l}\text { M, and perpendicular to an alternative horizontal plane (XY passing through the bilateral } \\
\text { A) }\end{array}$ \\
\hline
\end{tabular}

\footnotetext{
${ }^{\mathrm{a}}$ No metal markers used
} 
Fig. 1 a Linear measurements to the midsagittal plane. 1 maxillary rotation (point A-MSP), 2 maxillary dental midline deviation $\left(\mathrm{UI}_{\text {contact }}-\mathrm{MSP}\right), 3$ facial width (Zy-MSP), 4 maxillary width (J-MSP), 5 maxillary dental width (U6-MSP), 6 mandibular rotation (PogMSP), 7 mandibular dental midline deviation ( $\mathrm{LI}_{\text {contact }}-\mathrm{MSP}$ ), 8 mandibular width (Go-MSP). b Frontal view of a 3D volume rendering of a skull used for this study. The positions landmark configuration (green markers) is illustrated (also see Table 1)
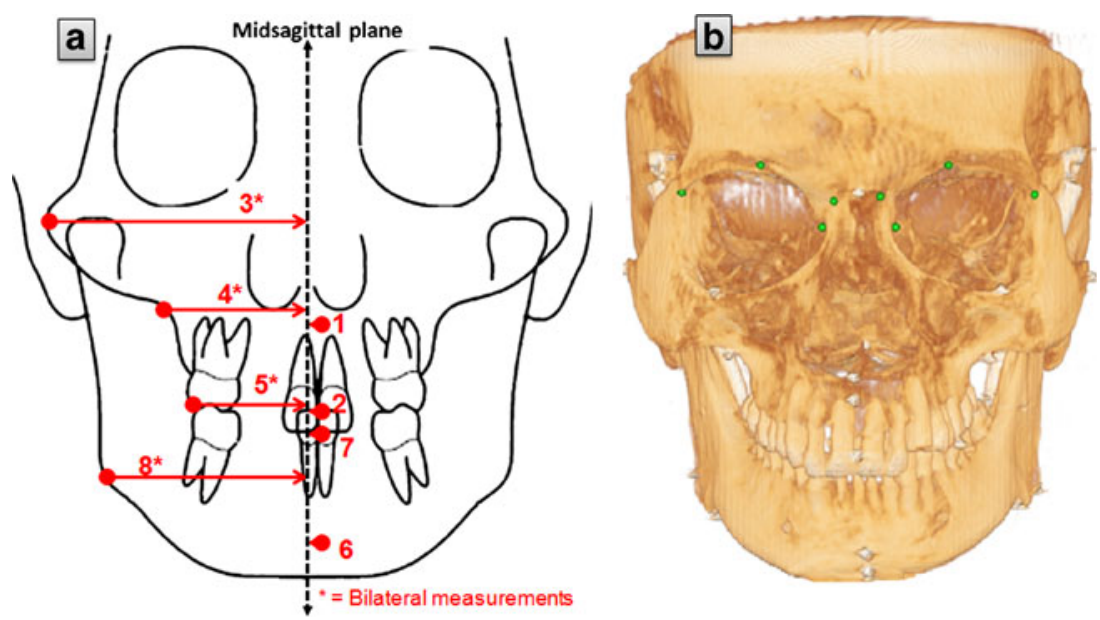

by connection of two midline structures perpendicular to a horizontal plane $(4,5$, and 6$)$. The software measured the distances illustrated in Fig. 1a by means of preprogrammed analyses. The distances were measured three times for each skull and the mean used as the reference value.

Morphometric method to determine the midsagittal plane

To determine the true midsagittal plane, the original and mirrored surface models were matched using partial Ordinary Procrustes Analysis (OPA) [16, 21]. After the hard-tissue surface models were segmented with the
SimPlant ${ }^{\circledR}$ Ortho software, digital markers with an $\varnothing$ of $2.4 \mathrm{~mm}$ were imported as STL files and placed in the landmark configuration position, which serves as reference for the OPA. Visible facial anatomical landmarks were used in the supraorbital and nasal bridge region (Table 1) that can be accurately identified by using the volume renderings and the cross-sectional slices in all three planes of the CBCT images. The infraorbital landmarks and zygomatic bones were not considered because they could be affected by midface deficiencies [22]. In addition, since most mandibular asymmetry and mild maxillary asymmetry are corrected with bilateral sagittal split osteotomies, genio-
Fig. 2 Morphometric method by means of OPA of original and mirrored $3 \mathrm{D}$ surface models used to derive the midsagittal plane

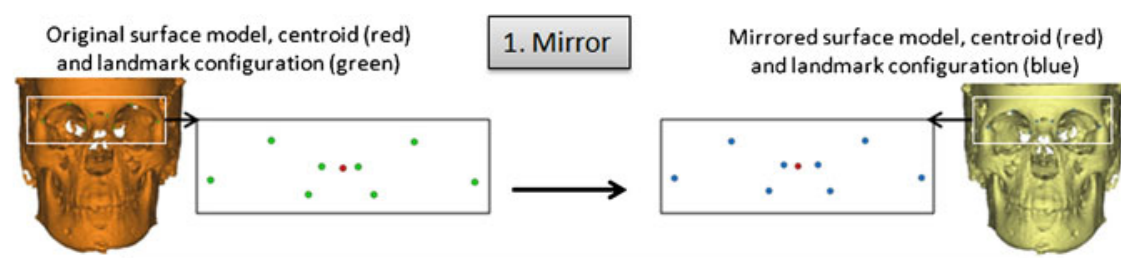

2. Shape alignment by means of partial Ordinary Procrustes Analysis

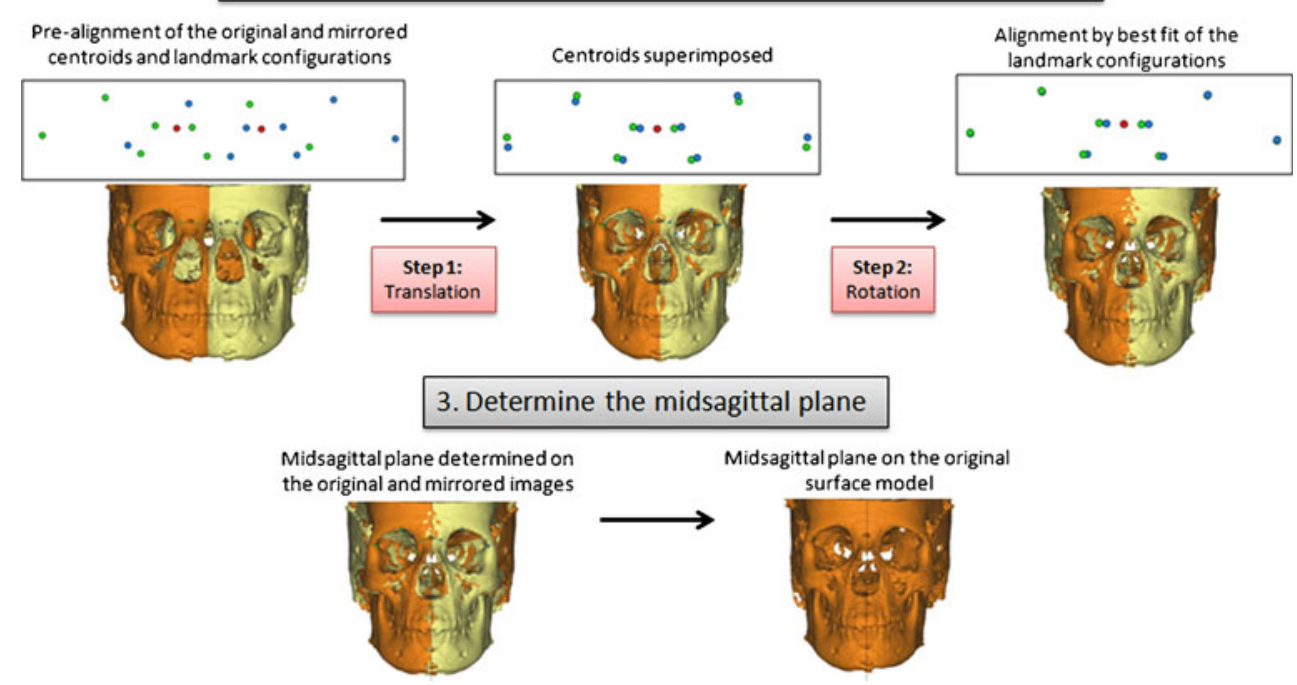


plasty, or Le Fort I surgery, the selected landmark configuration can be considered as stable reference marks for surgical planning [23] (Fig. 1b). The centroid positions were calculated with the software using the coordinate values of the landmark configuration. Mirror images of the original surface models, digital landmark configurations, and centroids were created around an arbitrary plane with the mirror tool of the software (Fig. 2). Shape alignment by means of partial OPA involves two steps: translation and rotation [21]. First, the centroid markers of the original and mirrored surface models were superimposed using the translation function of the software. Rotation of the mirrored landmark configuration around the geometric midpoint of the superimposed centroid markers followed until the best fit between all homologous landmarks were achieved by means of the least squared point distance while preserving the shape and size of each configuration [16] (Fig. 2). The individual symmetrical configuration of point
ANS, pogonion, and sella (S) was calculated as the mean of the original and mirrored landmarks. The software program created the midsagittal plane by constructing plane through the middle of these individual symmetrical configurations. Subsequently, the 12 linear distances illustrated in Fig. 1a were measured three times for each skull and the mean used as the reference value. In addition, the deviation from point $\mathrm{N}$ to the morphometric plane was also measured.

An error study was performed after a 3-week interval to validate the morphometric method. Wilcoxon signed-rank sum test was used to detect differences between the two morphometric midsagittal values. Agreement between the measurements was tested by the Pearson correlation coefficient. The standard error of measurement (SEM) of the repeated measurements was calculated as the square root of the variance of the random error from a two-way random effect ANOVA. The smallest detectable difference was then calculated as $1.96 \times \sqrt{2} \times$ SEM [24].
Table 2 Validity of the morphometric method used in this study

$P$ values of less than 0.05 considered to be significant. Mean, SD, and method error values in millimeters

$T$ session, SEM standard error of measurement, $S D D$ smallest detectable difference or method error at $95 \%$ confidence interval, $r$ intraobserver agreement, SD standard deviation

\begin{tabular}{|c|c|c|c|c|c|c|c|c|}
\hline \multirow[b]{2}{*}{ Variable } & \multicolumn{2}{|l|}{$\mathrm{T} 1$} & \multicolumn{2}{|l|}{$\mathrm{T} 2$} & \multicolumn{2}{|c|}{ Method error } & \multicolumn{2}{|c|}{ T1 vs. T2 } \\
\hline & Mean & $\mathrm{SD}( \pm)$ & Mean & $\mathrm{SD}( \pm)$ & SEM & SDD & $r$ & $P$ \\
\hline \multicolumn{9}{|l|}{ Asymmetric group $(n=5)$} \\
\hline Maxillary rotation & 1.38 & 0.73 & 1.56 & 0.55 & 0.07 & 0.21 & 0.904 & 0.60 \\
\hline Maxillary dental rotation & 1.25 & 0.81 & 1.36 & 1.05 & 0.10 & 0.28 & 0.930 & 0.92 \\
\hline Facial width left & 60.87 & 2.32 & 61.00 & 1.96 & 0.08 & 0.21 & 0.991 & 0.92 \\
\hline Facial width right & 64.24 & 1.06 & 63.83 & 1.03 & 0.25 & 0.70 & 0.895 & 0.47 \\
\hline Maxilla width left & 34.69 & 1.22 & 34.80 & 1.20 & 0.06 & 0.18 & 0.975 & 0.75 \\
\hline Maxilla width right & 38.21 & 1.62 & 38.01 & 1.89 & 0.08 & 0.23 & 0.988 & 0.60 \\
\hline Maxilla dental width left & 28.26 & 1.52 & 28.42 & 1.65 & 0.25 & 0.71 & 0.938 & 0.60 \\
\hline Maxilla dental width right & 28.51 & 2.70 & 28.38 & 3.08 & 0.16 & 0.44 & 0.989 & 0.75 \\
\hline Mandibula rotation & 7.02 & 5.32 & 7.42 & 5.06 & 0.16 & 0.45 & 0.998 & 0.92 \\
\hline Mandibula dental rotation & 1.89 & 2.18 & 2.09 & 2.61 & 0.17 & 0.48 & 0.984 & 0.75 \\
\hline Mandibula width left & 44.05 & 3.20 & 44.08 & 3.03 & 0.22 & 0.63 & 0.985 & 0.92 \\
\hline Mandibula width right & 44.66 & 1.53 & 44.47 & 1.97 & 0.24 & 0.67 & 0.954 & 0.75 \\
\hline Nasion deviation & 0.27 & 0.15 & 0.40 & 0.30 & 0.02 & 0.07 & 0.845 & 0.25 \\
\hline \multicolumn{9}{|l|}{ Symmetric group $(n=9)$} \\
\hline Maxillary rotation & 1.12 & 0.81 & 1.14 & 0.54 & 0.11 & 0.30 & 0.850 & 0.69 \\
\hline Maxillary dental rotation & 1.11 & 0.54 & 1.08 & 0.87 & 0.08 & 0.23 & 0.904 & 0.76 \\
\hline Facial width left & 58.12 & 4.09 & 58.08 & 3.85 & 0.06 & 0.17 & 0.998 & 0.90 \\
\hline Facial width right & 59.48 & 4.21 & 59.37 & 4.32 & 0.03 & 0.07 & 0.999 & 0.76 \\
\hline Maxilla width left & 35.50 & 2.55 & 35.31 & 2.49 & 0.11 & 0.30 & 0.984 & 0.97 \\
\hline Maxilla width right & 37.67 & 3.40 & 37.53 & 3.70 & 0.08 & 0.23 & 0.997 & 0.83 \\
\hline Maxilla dental width left & 25.72 & 2.00 & 25.73 & 1.86 & 0.12 & 0.32 & 0.982 & 0.97 \\
\hline Maxilla dental width right & 26.65 & 2.85 & 26.59 & 2.99 & 0.10 & 0.28 & 0.993 & 0.97 \\
\hline Mandibula rotation & 1.70 & 1.19 & 1.74 & 1.09 & 0.18 & 0.51 & 0.914 & 0.93 \\
\hline Mandibula dental rotation & 1.52 & 1.00 & 1.62 & 0.95 & 0.11 & 0.31 & 0.934 & 0.76 \\
\hline Mandibula width left & 43.31 & 4.46 & 43.44 & 4.34 & 0.25 & 0.68 & 0.993 & 0.97 \\
\hline Mandibula width right & 45.07 & 3.80 & 44.79 & 4.05 & 0.29 & 0.79 & 0.991 & 0.90 \\
\hline Nasion deviation & 0.55 & 0.27 & 0.33 & 0.26 & 0.02 & 0.06 & 0.871 & 0.93 \\
\hline
\end{tabular}


Comparison of the cephalometric midsagittal planes

The mean values and standard deviations were calculated for the 12 linear measurements of all the cephalometric 3D midsagittal planes. The mean of the repeated morphometric values of the error study was used as the reference values for comparison. The clinical accuracy of the cephalometric midsagittal planes was compared by means of the absolute error (AE). The AE was defined as the reference (morphometric) midsagittal plane values subtracted by the cephalometric midsagittal plane value. Additionally, MannWhitney tests were used to detect possible differences between the asymmetric and symmetric groups. $P$ values of less than 0.05 were considered significant. All statistics were performed with a standard statistical software package (SPSS version 16, Chicago, IL).

\section{Results}

The results of the error study (Table 2) validated the morphometric midsagittal plane and confirmed its accuracy and reliability previously reported $[15,18-20]$. There were no statistical differences between the measurements $(P=0.25$ $0.97)$, and the agreement was high $(r=0.845-0.999)$. The small method error (mean $=0.39 \mathrm{~mm} ; 95 \% \mathrm{CI}=0.31-$ $0.47 \mathrm{~mm}$ ) is more than clinically acceptable for cephalometric measurements and confirmed the accuracy of the method [25].

Table 3 The mean and standard deviation of the different midsagittal planes of the asymmetric and symmetric skulls

\begin{tabular}{|c|c|c|c|c|c|c|c|c|c|c|c|c|c|c|}
\hline \multirow[b]{4}{*}{ Variable } & \multirow{3}{*}{\multicolumn{2}{|c|}{$\begin{array}{l}\text { Morphometric } \\
\text { midsagittal plane }\end{array}$}} & \multicolumn{12}{|c|}{ Cephalometric midsagittal planes } \\
\hline & & & \multicolumn{6}{|c|}{ Three midline structures only } & \multicolumn{6}{|c|}{$\begin{array}{l}\text { Two midline structures perpendicular } \\
\text { to a horizontal plane }\end{array}$} \\
\hline & & & \multicolumn{2}{|l|}{1} & \multicolumn{2}{|l|}{2} & \multicolumn{2}{|l|}{3} & \multicolumn{2}{|l|}{4} & \multicolumn{2}{|l|}{5} & \multicolumn{2}{|l|}{6} \\
\hline & Mean & $\mathrm{SD}( \pm)$ & Mean & $\mathrm{SD}( \pm)$ & Mean & $\mathrm{SD}( \pm)$ & Mean & $\mathrm{SD}( \pm)$ & Mean & $\mathrm{SD}( \pm)$ & Mean & $\mathrm{SD}( \pm)$ & Mean & $\mathrm{SD}( \pm)$ \\
\hline \multicolumn{15}{|l|}{ Asymmetric group $(n=5)$} \\
\hline Maxillary rotation & 1.47 & 0.62 & 0.76 & 0.31 & 4.84 & 2.38 & 3.41 & 0.62 & 1.33 & 0.85 & 1.81 & 1.17 & 1.03 & 0.59 \\
\hline Maxillary dental rotation & 1.31 & 0.91 & 1.86 & 0.75 & 5.63 & 3.10 & 3.92 & 1.34 & 1.20 & 0.42 & 1.74 & 0.87 & 2.21 & 0.41 \\
\hline Facial width left & 60.94 & 2.14 & 62.15 & 1.57 & 60.94 & 3.27 & 61.26 & 1.64 & 61.65 & 1.87 & 62.12 & 1.85 & 62.13 & 1.79 \\
\hline Facial width right & 64.04 & 0.99 & 62.84 & 1.53 & 63.56 & 1.41 & 63.62 & 1.39 & 63.37 & 1.22 & 62.85 & 1.33 & 62.89 & 1.92 \\
\hline Maxilla width left & 34.75 & 1.20 & 36.50 & 1.11 & 33.80 & 3.83 & 33.71 & 1.17 & 35.17 & 1.13 & 35.18 & 1.22 & 36.15 & 1.59 \\
\hline Maxilla width right & 38.11 & 1.75 & 36.35 & 2.22 & 38.86 & 2.67 & 39.11 & 1.72 & 37.73 & 1.22 & 37.71 & 0.97 & 36.72 & 2.34 \\
\hline Maxilla dental width left & 28.34 & 1.54 & 31.06 & 1.86 & 26.82 & 6.68 & 26.54 & 2.48 & 28.89 & 2.45 & 28.83 & 2.77 & 30.21 & 2.65 \\
\hline Maxilla dental width right & 28.45 & 2.88 & 25.73 & 3.81 & 30.19 & 5.21 & 30.45 & 2.95 & 28.06 & 2.28 & 28.14 & 2.03 & 26.63 & 4.05 \\
\hline Mandibula rotation & 7.22 & 5.18 & 10.04 & 5.55 & 0.52 & 0.62 & 6.65 & 6.54 & 6.44 & 4.64 & 5.97 & 4.57 & 9.23 & 6.13 \\
\hline Mandibula dental rotation & 1.99 & 2.39 & 4.24 & 0.77 & 3.67 & 3.39 & 3.04 & 3.52 & 1.52 & 1.68 & 1.58 & 1.52 & 3.61 & 1.76 \\
\hline Mandibula width left & 44.06 & 3.09 & 47.71 & 3.18 & 43.62 & 6.98 & 43.22 & 2.78 & 45.47 & 2.64 & 46.26 & 2.65 & 46.40 & 2.92 \\
\hline Mandibula width right & 44.57 & 1.72 & 40.92 & 2.27 & 45.30 & 5.84 & 45.36 & 2.17 & 43.31 & 1.71 & 42.48 & 1.38 & 42.22 & 2.84 \\
\hline \multicolumn{15}{|l|}{ Symmetric group $(n=9)$} \\
\hline Maxillary rotation & 1.13 & 0.67 & 0.47 & 0.34 & 1.34 & 1.09 & 1.98 & 1.51 & 1.02 & 0.62 & 1.37 & 1.08 & 1.01 & 0.69 \\
\hline Maxillary dental rotation & 1.10 & 0.70 & 1.65 & 0.73 & 0.89 & 1.00 & 2.20 & 1.85 & 0.79 & 0.79 & 0.82 & 0.78 & 1.47 & 1.15 \\
\hline Facial width left & 58.10 & 3.85 & 59.31 & 4.35 & 58.77 & 4.34 & 58.56 & 4.00 & 58.85 & 4.37 & 58.64 & 4.30 & 59.11 & 4.22 \\
\hline Facial width right & 59.43 & 4.14 & 57.90 & 3.69 & 58.50 & 3.80 & 58.69 & 4.02 & 58.69 & 4.28 & 58.86 & 3.71 & 58.13 & 3.82 \\
\hline Maxilla width left & 35.41 & 2.45 & 36.78 & 3.34 & 35.40 & 3.10 & 35.13 & 2.44 & 35.62 & 3.36 & 35.58 & 2.99 & 36.36 & 3.16 \\
\hline Maxilla width right & 37.60 & 3.45 & 36.18 & 2.61 & 37.57 & 2.87 & 37.82 & 3.50 & 37.39 & 2.67 & 37.46 & 3.24 & 36.64 & 2.70 \\
\hline Maxilla dental width left & 25.73 & 1.87 & 27.72 & 2.12 & 25.54 & 2.00 & 25.09 & 1.20 & 25.73 & 1.92 & 25.80 & 2.30 & 26.59 & 1.55 \\
\hline Maxilla dental width right & 26.62 & 2.83 & 24.62 & 2.88 & 26.87 & 2.64 & 27.28 & 4.27 & 26.34 & 2.78 & 26.39 & 2.76 & 25.80 & 3.32 \\
\hline Mandibula rotation & 1.72 & 1.11 & 2.75 & 1.81 & 0.74 & 0.57 & 2.76 & 1.89 & 2.00 & 1.16 & 2.10 & 1.24 & 2.31 & 2.13 \\
\hline Mandibula dental rotation & 1.57 & 0.95 & 1.53 & 1.04 & 1.15 & 0.90 & 2.51 & 1.80 & 1.51 & 1.03 & 1.54 & 1.31 & 1.64 & 1.39 \\
\hline Mandibula width left & 43.37 & 4.27 & 45.35 & 4.48 & 44.43 & 5.08 & 43.80 & 3.64 & 43.76 & 4.58 & 44.28 & 4.34 & 44.67 & 4.39 \\
\hline Mandibula width right & 44.93 & 3.82 & 42.81 & 5.24 & 43.76 & 3.40 & 44.36 & 4.51 & 44.49 & 3.90 & 43.98 & 4.23 & 43.60 & 3.82 \\
\hline
\end{tabular}

All values in millimeters 
The mean and standard deviation of all the midsagittal planes are illustrated in Table 3. When comparing the cephalometric values to the morphometric values by means of the AE, a large variation was seen in both symmetrical and asymmetrical groups (Table 4). As expected, the midsagittal planes constructed by connecting midline structures (1-3) resembled the morphometric plane more closely in the symmetric group; however, the mean differences were still more than $1.00 \mathrm{~mm}$. In both groups, the values of the midsagittal planes (4 and 5) constructed as perpendiculars to the $\mathrm{FH}$ plane were clinically the most accurate in resembling the morphometric values. For most of the measurements of these planes, the mean absolute errors were less than $1.00 \mathrm{~mm}$. The differences between the values of midsagittal planes $1-3$ and 6 can be considered clinically relevant $(>1.00 \mathrm{~mm})$ compared to the morphological values in both groups.

The amount of mandibular rotation in the asymmetric group was $7.22 \pm 5.18 \mathrm{~mm}$ compared to $1.72 \pm 1.11 \mathrm{~mm}$ in the symmetric group. This was the only variable significantly different $(P=0.01)$ between the symmetric and asymmetric skulls. Further analysis of the asymmetric skulls revealed that the main causes of asymmetry were probably due to differences between the bilateral ramus and/or mandibular body lengths. There were clinically significant $(>1.00 \mathrm{~mm})$ differences between the ramus lengths only or the mandibular body lengths only in two skulls. In three skulls, clinically significant differences were found in both the ramus and mandibular body lengths.

Table 4 Clinical differences determined by means of the absolute error of the cephalometric midsagittal planes (1-6) when compared to the morphometric reference midsagittal plane

\begin{tabular}{|c|c|c|c|c|c|c|c|c|c|c|c|c|}
\hline \multirow[b]{4}{*}{ Variable } & \multicolumn{12}{|c|}{ Morphometric midsagittal plane vs. cephalometric midsagittal planes } \\
\hline & \multicolumn{6}{|c|}{ Three midline structures only } & \multicolumn{6}{|c|}{ Two midline structures perpendicular to a horizontal plane } \\
\hline & \multicolumn{2}{|l|}{1} & \multicolumn{2}{|l|}{2} & \multicolumn{2}{|l|}{3} & \multicolumn{2}{|l|}{4} & \multicolumn{2}{|l|}{5} & \multicolumn{2}{|l|}{6} \\
\hline & Mean & $\mathrm{SD}( \pm)$ & Mean & $\mathrm{SD}( \pm)$ & Mean & $\mathrm{SD}( \pm)$ & Mean & $\mathrm{SD}( \pm)$ & Mean & $\mathrm{SD}( \pm)$ & Mean & $\mathrm{SD}( \pm)$ \\
\hline \multicolumn{13}{|l|}{ Asymmetric group $(n=5)$} \\
\hline Maxillary rotation & 0.82 & 0.39 & 3.37 & 1.94 & 1.94 & 0.90 & 0.40 & 0.36 & 0.71 & 0.46 & 0.89 & 0.16 \\
\hline Maxillary dental rotation & 0.98 & 0.62 & 4.33 & 2.99 & 2.62 & 1.07 & 0.53 & 0.59 & 1.02 & 0.45 & 1.18 & 0.45 \\
\hline Facial width left & 1.21 & 0.63 & 1.06 & 1.02 & 0.73 & 0.26 & 0.87 & 0.28 & 1.28 & 0.70 & 1.20 & 0.93 \\
\hline Facial width right & 1.20 & 0.60 & 0.87 & 1.35 & 0.62 & 0.24 & 0.73 & 0.38 & 1.19 & 0.72 & 1.15 & 1.03 \\
\hline Maxilla width left & 1.75 & 0.77 & 2.89 & 1.84 & 1.11 & 0.69 & 0.53 & 0.36 & 0.69 & 0.53 & 1.62 & 1.05 \\
\hline Maxilla width right & 1.76 & 0.87 & 2.85 & 1.85 & 1.07 & 0.70 & 0.50 & 0.44 & 0.66 & 0.61 & 1.70 & 1.01 \\
\hline Maxilla dental width left & 2.72 & 1.22 & 4.76 & 3.09 & 1.80 & 1.33 & 0.84 & 0.73 & 1.00 & 0.95 & 2.25 & 1.48 \\
\hline Maxilla dental width right & 2.72 & 1.39 & 4.97 & 3.25 & 2.00 & 1.07 & 0.58 & 0.67 & 0.70 & 0.84 & 2.20 & 1.48 \\
\hline Mandibula rotation & 3.85 & 1.62 & 6.70 & 5.38 & 2.44 & 1.06 & 0.85 & 1.30 & 1.30 & 1.14 & 3.03 & 1.80 \\
\hline Mandibula dental rotation & 2.76 & 1.39 & 3.36 & 3.47 & 1.38 & 1.22 & 0.54 & 0.69 & 0.62 & 0.72 & 2.42 & 1.55 \\
\hline Mandibula width left & 3.65 & 1.06 & 4.71 & 4.74 & 0.98 & 1.19 & 1.47 & 1.12 & 2.20 & 1.41 & 2.34 & 1.36 \\
\hline Mandibula width right & 3.65 & 1.17 & 4.63 & 4.19 & 0.83 & 1.06 & 1.31 & 1.07 & 2.09 & 1.36 & 2.34 & 1.47 \\
\hline \multicolumn{13}{|l|}{ Symmetric group $(n=9)$} \\
\hline Maxillary rotation & 0.75 & 0.65 & 1.11 & 0.67 & 1.23 & 1.19 & 0.70 & 0.40 & 0.72 & 0.50 & 0.78 & 0.62 \\
\hline Maxillary dental rotation & 1.27 & 0.37 & 0.98 & 0.76 & 1.48 & 1.42 & 0.56 & 0.49 & 0.69 & 0.36 & 1.11 & 0.83 \\
\hline Facial width left & 1.38 & 0.93 & 0.99 & 0.89 & 0.69 & 0.68 & 0.99 & 0.90 & 0.96 & 0.88 & 1.41 & 1.21 \\
\hline Facial width right & 1.52 & 1.15 & 1.07 & 1.02 & 0.81 & 0.88 & 0.92 & 0.68 & 0.93 & 0.83 & 1.49 & 1.26 \\
\hline Maxilla width left & 1.52 & 0.81 & 0.95 & 0.55 & 1.00 & 0.73 & 0.85 & 0.69 & 0.78 & 0.60 & 1.13 & 0.94 \\
\hline Maxilla width right & 1.65 & 0.79 & 0.96 & 0.56 & 0.99 & 0.71 & 0.82 & 0.75 & 0.63 & 0.65 & 1.15 & 0.90 \\
\hline Maxilla dental width left & 2.17 & 1.03 & 1.22 & 0.73 & 1.64 & 1.13 & 0.80 & 0.71 & 0.72 & 0.32 & 1.21 & 0.85 \\
\hline Maxilla dental width right & 2.60 & 0.83 & 1.38 & 1.05 & 1.97 & 1.39 & 0.65 & 0.49 & 0.71 & 0.37 & 1.58 & 0.86 \\
\hline Mandibula rotation & 1.77 & 1.13 & 1.09 & 0.88 & 2.02 & 1.61 & 1.00 & 0.62 & 0.88 & 0.57 & 2.03 & 1.11 \\
\hline Mandibula dental rotation & 1.48 & 0.75 & 0.99 & 0.65 & 1.29 & 1.15 & 0.73 & 0.55 & 0.76 & 0.35 & 1.49 & 0.93 \\
\hline Mandibula width left & 2.42 & 1.17 & 1.88 & 1.48 & 1.32 & 0.97 & 0.91 & 0.50 & 1.24 & 0.82 & 1.30 & 0.91 \\
\hline Mandibula width right & 2.48 & 1.34 & 1.96 & 1.63 & 1.41 & 0.99 & 0.90 & 0.45 & 1.22 & 0.78 & 1.33 & 0.87 \\
\hline
\end{tabular}

All values in millimeters 


\section{Discussion}

The results of this study show that there were clinically relevant differences between cephalometric midsagittal planes and the true plane of symmetry on CBCT images. Therefore, the additional cost of extra software to extract a morphometric midsagittal plane might outweigh the risk of misdiagnosis or inaccurate treatment planning of craniofacial asymmetry. In addition, other advantages of a morphometric midsagittal plane further justify its use. A major advantage is that it can compute the midsagittal plane using intact regions unaffected by the asymmetry $[16,19,20]$ and therefore can be used to determine the midsagittal plane in severe and congenital asymmetries. This is not always possible when using cephalometric planes based on anatomical landmarks, i.e., the auditory meatus might be affected in congenital malformations which would make the Frankfort horizontal plane unreliable. Other advantages of a midsagittal plane computed with morphometric methods are: it is very reliable, it can be simple and quick (newer software can automatically extract the midsagittal plane), it can be applied to 3D datasets from laser surface scanners and stereophotogrammetry, and all to computerized tomography imaging $[15,16,18-20]$.

We used dry skulls and metal markers aiming to reduce the measurement error. Although the asymmetric sample size can be regarded as small, it can be justified because the method error was very small and able to detect true clinical differences between the different midsagittal planes. In addition, it must be noted that the sample is unique and that asymmetric dry skulls are difficult to acquire for comparative study. Jacobsen et al. [26] defined the 3D midsagittal plane as a midline plane bisecting the head sagittally through point $\mathrm{N}$ when viewing the patient in natural head position (NHP) from the frontal view. Therefore, the midsagittal plane derived from the NHP is based on the visual perception and does not rely on internal structures. In the present study, 3D reference planes based on the NHP could not be investigated because dry skulls were used. However, we found that the mean deviation of point $\mathrm{N}$ from the morphometric midsagittal plane was less than $0.50 \mathrm{~mm}$ in both groups suggesting its suitability as reference point in both groups. Future research may investigate if the NHP is more suitable and reliable than the anatomical landmarks for the construction of standardized planes for cephalometric analysis with $\mathrm{CBCT}$ imaging.

Trpkova et al. [27] studied two-dimensional radiographs of various asymmetries and concluded that vertical lines constructed as perpendiculars through midpoints between bilateral pairs of orbital landmarks are more accurate and valid than those constructed between two midpoints. Although we studied 3D images, the results of the present study are in agreement with this conclusion. The values from midsagittal planes constructed as perpendiculars to the FH plane showed the least differences from the morphometric values. The midsagittal plane constructed as a perpendicular to the $\mathrm{FH}$ plane passing through $\mathrm{N}$ and $\mathrm{S}$ mimicked the morphometric plane the best in both groups. However, care should be taken to apply these data for clinical use. Although the mean differences between this plane and the morphometric plane were less than $1.00 \mathrm{~mm}$ for most measurements, the standard deviation was often large and significant [25]. In addition, our sample did not include congenital asymmetries or asymmetries of the midface. This is important as the FH plane might be invalid as reference plane for congenital asymmetries and asymmetries associated with midface deficiencies.

Recently, a standardized plane orientation for 3D cephalometric analysis was used to describe asymmetry
Fig. 3 a Midsagittal plane constructed connecting landmarks MDFM and ELSA perpendicular to the horizontal plane XY. b A small lateral deviation $(0.40 \mathrm{~mm})$ in MDFM results in significantly greater the linear measurement error when measuring Pog to the midsagittal plane $(6.15 \mathrm{~mm}$ difference)
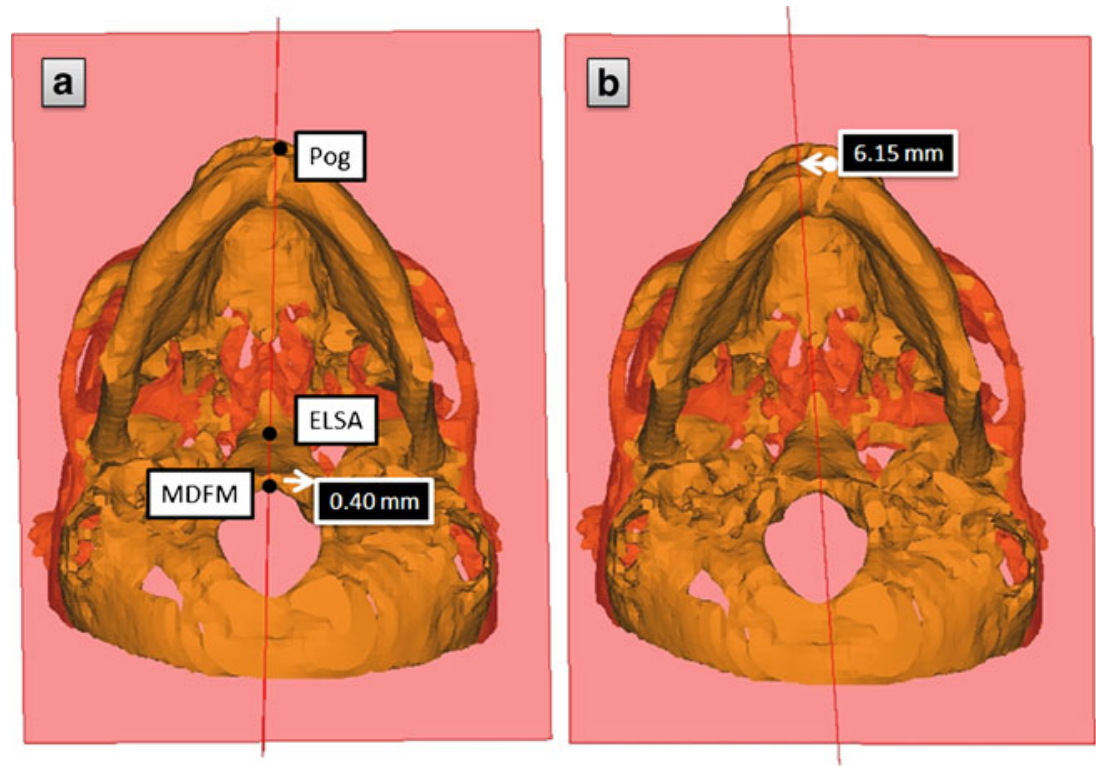
$[4,28]$. When using these planes, the effect of the positioning of the patients head during image acquisition is eliminated. The landmarks used to construct the planes were shown to be very reproducible, and all points are located on sutures that are not significantly affected by growth after 5 years of age [4, 28]. Yàñez-Vico et al. [4] reported an intraobserver error of $1.36 \mathrm{~mm}$ for linear measurements and $0.91^{\circ}$ for angular measurements using this plane orientation. However, it must be noted that their measurements were not made to the midsagittal plane but derived from the subtraction of left and right side measurements. Importantly, we found that a geometric principal described by Nagasaka et al. [29] has a significant effect on the measurements to the midsagittal plane. This principal illustrated that the distances between landmarks also have an influence on the magnitude of measuring error of the linear and angular measurements and that the more close two landmarks are, the greater the angular measurement error tends to be. This geometric principal has a significant $3 \mathrm{D}$ effect on the construction of the midsagittal plane because the landmarks of foramen spinosum midpoint (ELSA) and MDFM are very close to each other. Therefore, a $0.40-\mathrm{mm}$ deviation of MDFM could result in $6.15-\mathrm{mm}$ difference of the variable used to measure mandibular deviation in one of the skulls (Fig. 3). Although the landmarks are situated on sutures and not affected by growth after 5 years of age, differences in remodeling occur which may result in small asymmetries [30]. Therefore, the midsagittal plane of this "standardized" plane orientation might not be valid for asymmetry analysis.

The large variations of the cephalometric midsagittal planes in the symmetric group were somewhat surprising but can possibly be explained by the fact that a perfect "symmetric" face does not really exist and a degree of facial asymmetry is inherent for every individual $[1,12$, 13]. Therefore, midline landmarks used to construct 3D midsagittal planes are also likely to deviate from the true plane of symmetry. Moreover, the combined 3D effect when connecting two or more of the off-center midline points resulting from small regional remodeling could produce a significant deviation from the actual plane of symmetry. This supports the suggestion that internal structures of the skull may be irrelevant to the visible facial symmetry, even in symmetric skulls. Therefore, determining an absolute midsagittal plane based on midline cephalometric points will vary among individuals and remains questionable.

\section{Conclusion}

Clinical relevant differences exist between the different 3D cephalometric midsagittal planes and the true plane of symmetry determined by the visible facial features. When using the cephalometric planes based on midline structures for clinical diagnosis and treatment planning of craniofacial asymmetry, care has to be taken as they might differ from the true plane of symmetry. A morphometric approach to determine the midsagittal plane using visually intact regions of the skull not affected by the asymmetry as the reference might be more valuable for diagnosis and treatment planning of craniofacial asymmetry.

Conflict of interest The authors declare that they have no conflict of interest.

Authors' contributions Dr. Damstra and Dr. Fourie contributed to all stages of the research and manuscript preparation. M De Wit contributed to the study design and produced the digital markers. Dr Ren contributed to the study design and preparation of the manuscript.

Open Access This article is distributed under the terms of the Creative Commons Attribution Noncommercial License which permits any noncommercial use, distribution, and reproduction in any medium, provided the original author(s) and source are credited.

\section{References}

1. Klingenberg CP, Barluenga M, Meyer A (2002) Shape analysis of symmetric structures: quantifying variation among individuals and asymmetry. Evolution 56:1909-1920

2. Profitt WR, Turvey TA (1991) Dentofacial asymmetry. In: Profitt WR, White RP Jr (eds) Surgical orthodontic treatment. Mosby, St Louis, pp 483-549

3. Hwang H-S, Hwang CH, Lee K-H, Kang B-C (2006) Maxillofacial 3-dimensional image analysis for the diagnosis of facial asymmetry. Am J Orthod Dentofacial Orthop 130:779-785

4. Yàñez-Vico RM, Iglesias-Linares A, Torres-Lagares D, GurierrezPerez JL, Solano-Reina E (2010) Three-dimensional evaluation of craniofacial asymmetry: an analysis using computed tomography. Clin Oral Investig. doi:10.1007/s00784-010-0441-7

5. Tuncer BB, Atac MS, Yuksel S (2009) A case report comparing $3-\mathrm{D}$ evaluation in the diagnosis and treatment planning of hemimandibular hyperplasia with conventional radiography. J Craniomaxillofac Surg. doi:10.1016/j.jcms.2009.01.004

6. Baek S-K, Cho I-S, Chang Y-I, Kim M-J (2007) Skeletodental factors affecting chin point deviation in female patients with class III malocclusion and facial asymmetry: a three dimensional analysis using computed tomography. Oral Surg Oral Med Oral Pathol Oral Radiol Endod 104:628-39

7. Kwon T-G, Park H-S, Ryoo H-M, Lee S-H (2006) A comparison of craniofacial morphology in patients with and without facial asymmetry - a three-dimensional analysis with computed tomography. Int J Oral Maxillofac Surg 35:43-48

8. Junck L, Moen JG, Hutchins GD, Brown MB, Kuhl DE (1990) Correlation methods for the centering, rotation and alignment of functional brain images. J Nucl Med 31:1220-1226

9. Lui Y, Collins RT, Rothfus WE (2001) Robust midsagittal plane extraction from normal and pathological 3-D neuroradiology images. IEEE Trans Med Imaging 20:175-192 
10. Swennen GJR, Schutyser F, Hausamne JE (2005) Threedimensional cephalometry. A color atlas and manual. Heidelberg Springer, Berlin, pp 183-226

11. Damstra J, Oosterkamp BCM, Jansma J, Ren Y (2010) Combined 3-dimensional and mirror-image analysis for the diagnosis for asymmetry. Accepted for publication, Am J Orthod Dentofacial Orthop

12. Gawilkowska A, Szczurwski J, Czerwinski F, Miklaszwenska D, Adamiec A, Dzieciolowska E (2007) The fluctuating asymmetry of mediaeval and modern human skulls. Homo 58:159-172

13. Haraguchi S, Iguchi Y, Takada K (2008) Asymmetry of the face in orthodontic patients. Angle Orthod 78:421-426

14. Ferrario VF, Sforsa C, Poggio CE, Tartaglia G (1994) Distance from symmetry: a three-dimensional evaluation of facial asymmetry. J Oral Maxillofac Surg 52:1126-1132

15. De Momi E, Chapuis J, Pappas I, Ferrigno G, Hallerman W, Schramm A, Caversaccio M (2006) Automatic extraction of the mid-facial plane for craniomaxillofacial surgery planning. Int $\mathrm{J}$ Oral Maxillofac Surg 35:636-642

16. Hajeer MY, Ayoub AF, Millet DT (2004) Three-dimensional assessment of facial soft-tissue asymmetry before and after orthognathic surgery. Br J Oral Maxillofac Surg 42:396-404

17. McIntyre GT, Mossey PA (2003) Size and shape measurement in contemporary cephalometrics. Eur J Orthod 25:231-242

18. Hartman J, Meyer-Marcotty P, Hausler G, Stellzig-Eisenhauer A (2007) Reliability of a method for computing facial symmetry plane and degree of asymmetry based on 3D-data. J Orofac Orthop 68:477-490

19. Benz M, Laboureux X, Maier T, Nkenke E, Seeger S, Neukam FW et al (2002) The symmetry of faces. In: Greiner G, Niemann H, Ertl T, Girod B, Seidel HP (eds) Vision, modeling, and visualization. IOS, Amsterdam, pp 332-339

20. Meyer-Marcotty P, Alpers GW, Gerdes ABM, Stellzig-Eisenhauer A (2010) Impact of facial asymmetry in visual perception: a 3dimensional data analysis. Am J Orthod Dentofacial Orthop 137:168.e-168.e8
21. Dryden IL, Mardia KV (1998) Statistical shape analysis. Wiley, Chichester, pp 45-47

22. Moro A, Correra P, Boniello R, Gaspari G, Pelo S (2009) Threedimensional analysis in facial asymmetry: comparison with model analysis and conventional two-dimensional analysis. J Craniofac Surg 20:417-422

23. Reyneke JP (2003) Basic guidelines for the diagnosis and treatment of specific dentofacial deformities. In: Reyneke JP (ed) Essentials of orthognathic surgery. Quintessence Publishing Co Inc, Hanover Park, pp 230-245

24. Damstra J, Fourie Z, Huddleston Slater JJ, Ren Y (2010) Accuracy of linear measurements from cone-beam computed tomography-derived surface models of different voxel sizes. Am J Orthod Dentofacial Orthop 137:16.e.1-16.e.6

25. Richardson A (1981) A comparison of traditional and computerized methods of cephalometric analysis. Eur J Orthod 3:15-20

26. Jacobson RL (2006) Three-dimensional cephalometry. In: Jacobson A, Jacobson RL (eds) Radiographic cephalometry: from basics to 3D imaging, 2nd edn. Quintessence Publishing Co Inc, Hanover Park, pp 233-47

27. Trpkova B, Prasad NG, Lam EWN, Raboud D, Glover KE, Major PW (2003) Assessment of facial asymmetries from posterior cephalograms: validity of reference lines. Am J Orthod Dentofacial Orthop 123:512-520

28. Lagravere MO, Hansen L, Harzer W, Major PW (2006) Plane orientation for standardization in 3-dimensional cephalometric analysis with computerized tomography imaging. Am J Orthod Dentofacial Orthop 129:601-604

29. Nagasaka S, Fujimora T, Segoshi K (2003) Development of a non-radiographic cephalometric system. Eur J Orthod 25:7785

30. Kim YH, Sato K, Mitani H, Shimizu Y, Kikuchi M (2003) Asymmetry of the sphenoid bone and its suitability as a reference for analysing craniofacial asymmetry. Am J Orthod Dentofacial Orthop 124:656-662 